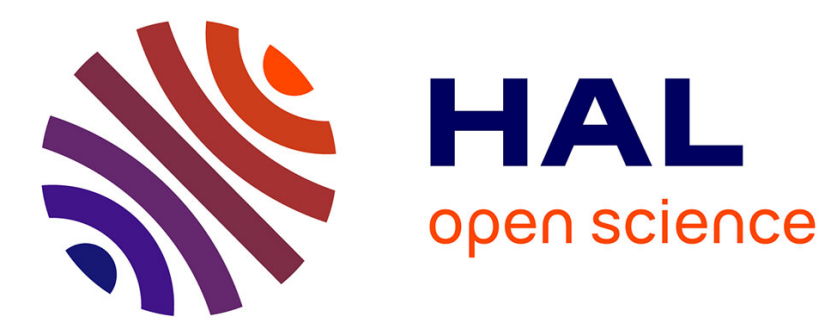

\title{
Unravelling the Mechanism of RNA-Polymerase Forward Motion by Using Mechanical Force
}

Philippe Thomen, Pascal J. Lopez, François Heslot

\section{To cite this version:}

Philippe Thomen, Pascal J. Lopez, François Heslot. Unravelling the Mechanism of RNA-Polymerase Forward Motion by Using Mechanical Force. Physical Review Letters, 2005, 94, pp.128102. hal00017065

\section{HAL Id: hal-00017065 \\ https://hal.science/hal-00017065}

Submitted on 16 Jan 2006

HAL is a multi-disciplinary open access archive for the deposit and dissemination of scientific research documents, whether they are published or not. The documents may come from teaching and research institutions in France or abroad, or from public or private research centers.
L'archive ouverte pluridisciplinaire HAL, est destinée au dépôt et à la diffusion de documents scientifiques de niveau recherche, publiés ou non, émanant des établissements d'enseignement et de recherche français ou étrangers, des laboratoires publics ou privés. 


\title{
Unravelling the mechanism of RNA-polymerase forward motion by using mechanical force
}

\author{
Philippe Thomen, Pascal J. Lopez ${ }^{\ddagger}$, François Heslot* \\ Laboratoire Pierre Aigrain, Ecole Normale Supérieure, 24 rue Lhomond, 75005 Paris, France \\ ¥ Laboratoire Organismes Photosynthétiques et Environnement, \\ Ecole Normale Supérieure, 46 rue d'Ulm, 75005 Paris, France
}

(Dated: December 16, 2004)

\begin{abstract}
Polymerases form a class of enzymes that act as molecular motors as they move along their nucleic acid substrate during catalysis, incorporating nucleotide triphosphates at the end of the growing chain and consuming chemical energy. A debated issue is how the enzyme converts chemical energy into motion [1]. In a single molecule assay, we studied how an opposing mechanical force affects the translocation rate of T7 RNA-polymerase. Our measurements show that force acts as a competitive inhibitor of nucleotide binding. This result is interpreted in the context of possible models, and with respect to published crystal structures of T7 RNA polymerase. The transcribing complex appears to utilize only a small fraction of the energy of hydrolysis to perform mechanical work, with the remainder being converted to heat.
\end{abstract}

PACS numbers: 87.14.Gg, 87.15.-v, 87.15.La, 87.80.Cc, 87.15.He

Keywords: DNA, single-molecule

Polymerases form a class of essential enzymes that carry out transcription, replication or repair of nucleic acids. RNA polymerases (RNAP) carry out an essential step in gene expression, the synthesis of a RNA copy from the DNA template. T7 RNAP, a single unit polymerase with known crystal structures $[2-5]$, is a prototype of this protein class. After stages of promoter recognition and initial transcription of a few bases, conformational changes occur [2, 3], allowing the protein to enter elongation mode [6] during which synthesis of RNA occurs by processive motion of RNAP along the DNA template. The standard textbook description of transcription is that the motor is "powered" by the chemical energy of NTP hydrolysis (see [7] for an estimation of this energy). In order to examine this view, we have performed single molecule measurements to study the influence of mechanical force on the kinetic properties of $\mathrm{T} 7$ RNAP transcription.

In the present work the elongation mode of the T7 RNAP is investigated. The configuration is presented in Fig. 1. For the experiments presented here, the concentration $\mathrm{C}_{0}$ of ATP, GTP, CTP and UTP was identical for each nucleotide and ranged from about $30 \mu \mathrm{M}$ up to 590 $\mu \mathrm{M}$. The measurements are performed using a feedback mode, where the applied force $\mathrm{F}$ is maintained constant. The force $\mathrm{F}$ ranged from $5 \mathrm{pN}$ to $15 \mathrm{pN}$.

Fig. 2 shows examples of measurements performed with different NTP concentrations at $5 \mathrm{pN}$. We measured over at least 8 seconds the extension of DNA vs. time for a given enzyme at a given force, and calculated the average velocity $\mathrm{V}$ by a linear fit. Within the apparatus accuracy and within this time scale, we only very seldomly observed pauses and excluded the few unambiguous pauses encountered for velocity determination [12]. T7 RNAP appears to behave differently in this respect than the Escherichia coli enzyme, for which the occurrence of many pauses in the range 1 to hundreds of seconds has been documented [13]. We observe a large variability in the measured velocities (estimated standard deviation: $30 \%$ ). A high variability is a common feature of single molecule experiments [14]. In the force range studied, we have not observed stalling, i.e. a smooth lowering of velocity vs. force, with stall occurring when the force is raised further. Reversible and irreversible stalling has been reported for E.coli RNAP in a range of forces above $20 \mathrm{pN}$. With the T7 RNAP however, we observe that the enzyme under force may abruptly stop transcribing. This may be irreversible, or in some cases a reversible event i.e. polymerization resumes when the force is lowered (unpublished).

We analyze the influence of force on the enzyme under steady-state transcription elongation conditions. Because it is convenient to analyze enzyme kinetics, we present the data using the Lineweaver-Burke plot where $1 /\langle V\rangle$ (in second per base, related to the time taken by the enzyme to perform a catalytic cycle) is plotted as a function of $1 / \mathrm{C}_{0}$ (related to the time taken for the catalytic site of the enzyme to encounter a nucleotide). Fig. 3 shows the $1 /\langle V\rangle$ vs. $1 / \mathrm{C}_{0}$ plots at different force levels. At low $1 / \mathrm{C}_{0}$ (i.e. large $\mathrm{C}_{0}$ ), nucleotide binding is not limiting the kinetics and the velocity is thus limited by some intrinsic minimal time lapse necessary for the enzyme to perform a catalytic cycle (this time is $\delta / \mathrm{V}_{\max }$, where $\mathrm{V}_{\max }$ is the maximum velocity of the enzyme and $\delta$ is the enzyme step per cycle assumed to be of one base pair). In this regime, the force is observed to have no significant effect on the average velocity. At large $1 / \mathrm{C}_{0}$ (i.e. small $\mathrm{C}_{0}$ ), the velocity is limited by the availability of nucleotides and their binding kinetics. In this regime, we observe that the effect of force is to lower the average velocity. The data are well fit by lines, (excluding the points at the lowest concentration [15]), a feature com- 
patible with standard Michaelis-Menten kinetics. The fitted lines have identical $\mathrm{Y}$-intercepts (identical $\mathrm{V}_{\max }$ ) but varying slopes (corresponding to $\mathrm{K}_{M} / \mathrm{V}_{\max }$, with $\mathrm{K}_{M}$ being the apparent nucleotide binding constant). This reveals that the applied load acts as a competitive inhibitor of NTP binding: the effect of an increasing force is an increase in $\mathrm{K}_{M}$ but $\mathrm{V}_{\max }$ stays constant.

One would like now to interpret why force acts as a competitive inhibitor, somehow inhibiting NTP binding. One might propose that application of force inhibits a conformational change in the polymerase that is required for NTP binding to occur. While it has been proposed that NTP binding is associated with a conformational change in the RNAP, there is a priori no justification for believing that application of force in a manner depicted in Fig. 1 would either favor or disfavor such a conformational change. On the other hand, it is easy to see how the application of force would inhibit forward motion and thus inhibit NTP binding: the polymerase must move forward after completion of the bond formation step to clear the 3' end of the RNA out of the binding site to make room for the next incoming NTP. For this

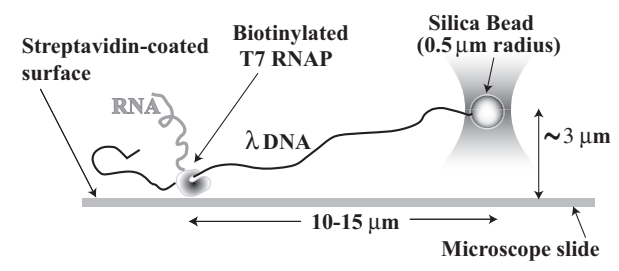

FIG. 1: Experimental configuration: the DNA template is lambda phage DNA (48502 base pairs, about $16 \mu \mathrm{m}$ in length) in which a T7 promoter is introduced at one end [9]. The other end of the DNA carries a digoxygenin and is attached to a microscopic bead coated with an antibody directed against digoxygenin. The transcribing polymerase is biotinylated at its N-terminus [10] and binds to a streptavidin coated solid surface. To detect tethered beads a flow is created. After selecting a tethered bead under the microscope, the 4 types of nucleotide are added to the sample. The enzyme pulls the bead as it transcribes the DNA, thereby shortening the tether. The bead is held in an optical tweezers equipped with force measurement [11]. Measurements are performed in a feedback mode where a constant force can be maintained on the DNA: the voltage corresponding to the measured force is fed back to a piezo-electric translation stage that laterally displaces the sample while the trap position is fixed. In this mode, the translocation of the enzyme along DNA is reflected by the displacement of the sample. The electronic time constant of the force feedback loop is $20 \mathrm{~ms}$. The position and force data are filtered through an antialiasing filter at $44 \mathrm{~Hz}$, sampled with 16 bit analog to digital conversion at a rate of $100 \mathrm{~Hz}$ and are stored onto a hard disk. The buffer, sample holder and the objective are temperature regulated at $27^{\circ} \mathrm{C}$. In order to avoid potential photodamage of the polymerase by the optical tweezers, we have taken the precaution of preventing the laser from impinging on the enzyme. This is possible because of the length of the DNA template. motion, the enzyme performs mechanical work against a load. A simple model using a classic kinetic description in which the enzyme transits stochastically through a sequence of states has been proposed by Guajardo and Sousa [17]. It consists of decomposing the binding step into two substates: (1) after completion of the bond formation step the polymerase is able to move forward by random thermal motion even against a load; then (2) an incoming NTP binds. This is followed by hydrolysis (3) but the corresponding liberated energy produces heat rather than mechanical work. Step (3) is assumed to be irreversible, so that no backwards motion is possible. Such a model predicts that force should be a competitive inhibitor of nucleotide binding. The corresponding scheme is described in Fig. 4a. Provided that the step following binding is slow with respect to $\mathrm{k}_{-2}$, the expression for $\mathrm{K}_{M}$ given by this model is: $\mathrm{K}_{M}=\mathrm{K}_{\text {diss }}(1+$ $\mathrm{k}_{-1} / \mathrm{k}_{1}$ ), with $\mathrm{K}_{\text {diss }}=\mathrm{k}_{-2} / \mathrm{k}_{2}$. Assuming that the force $\mathrm{F}$ acts essentially on the translocation step, i.e. on the $\mathrm{k}_{-1}$ and $\mathrm{k}_{1}$ constants, one gets: $\mathrm{K}_{M}(\mathrm{~F})=\mathrm{K}_{\text {diss }}[1+$ $\left.\mathrm{K} \exp \left(\mathrm{F}^{*} \delta / \mathrm{kT}\right)\right]$, where $\delta$ is the enzyme step size. The experimental $\mathrm{K}_{M}$ values are obtained from linear fits in Fig. 3, giving $\mathrm{K}=0.27$ and $\mathrm{K}_{\text {diss }}=124 \mu \mathrm{M}$ (see Fig. 3 inset). The corresponding free energy difference $\Delta \mathrm{G}_{0}$ for the forward motion is $\mathrm{kT} \ln (\mathrm{K})=-1.3 \mathrm{kT}$. Our data support a view approaching that proposed by Guajardo and Souza, although in their model they had $\Delta \mathrm{G}_{0} \geqslant 0$. The corresponding "flashing ratchet" or "two state model" Brownian motor (see [19] and references therein) considers that a Brownian particle can undergo directional movement by alternating between two states: in state 1 the particle essentially pinned and in state 2 (flat potential landscape [20]) diffusion can occur. However, $\Delta \mathrm{G}_{0}<0$ entails that $\mathrm{E}_{\text {post }}$ is energetically favored with respect to

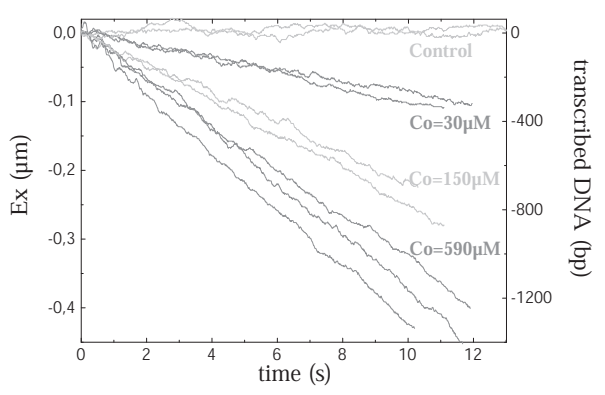

FIG. 2: Examples of experimental recordings: the measurements where performed at forces near $5 \mathrm{pN}$. Extension (Ex), i.e. the distance between the polymerase and the bead, is plotted vs. time. Transcription is revealed by the decrease of Ex. For each curve, extension and time have been shifted for comparison. Each curve corresponds to a different polymerase, the NTP concentrations are indicated on the figure. As a control we plot two Ex(t) curves obtained with a simple DNA construction attached between the surface and the bead. The average velocity of the enzyme (over at least 8 seconds) is extracted from those curves. 
$\mathrm{E}_{\text {pre }}$, i.e. the potential is not flat.

Crystallographic structures of the T7 RNA polymerase have been obtained with various substrates, yielding four different structures. Those structures may be interpreted as snapshots of various states of the enzyme during the catalytic cycle, and appear in Fig. 4b (in bold face). The structural data suggest that the post-translocated state $\mathrm{E}_{\text {post }}$, where the enzyme is waiting for NTP, is a stable state. The negative estimate of $\Delta \mathrm{G}_{0}$ (the free energy difference between $\mathrm{E}_{\text {pre }}$ and $\mathrm{E}_{\text {post }}$ in our model) is consistent with this. The crystal data also indicate that neither movement of the enzyme nor significant conformational change occur upon PPi cleavage. Force being a competitive inhibitor is also consistent with this finding. From structural analysis, it has been argued that translocation occurs between two states: $\mathrm{E}_{\text {pre }}: \mathrm{PPi}$ where the enzyme is pre-translocated with PPi bound, and $\mathrm{E}_{\text {post }}$, the enzyme post-translocated waiting for NTP. We propose a mechanism of RNA polymerase which is consistent with published crystal structures, but differs from the

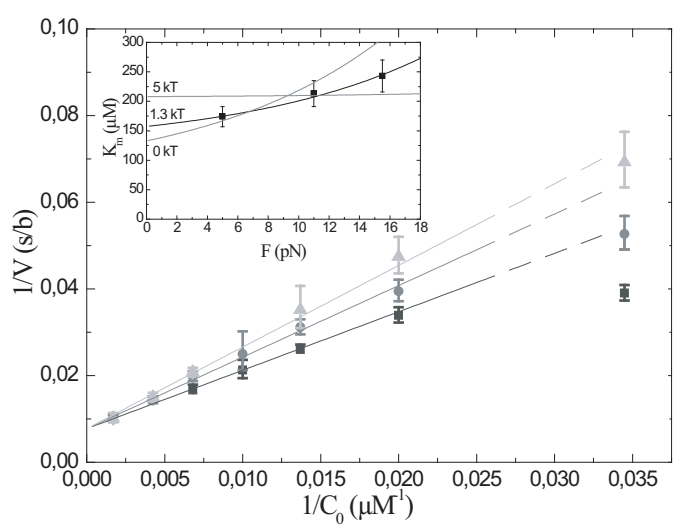

FIG. 3: Lineweaver-Burke plot of the experimental data: the inverse of the average velocity $1 /\langle V\rangle$ is plotted as a function of $1 / \mathrm{C}_{0}$ for three forces: $5 \pm 1 \mathrm{pN}$ (squares), 11 $\pm 0.7 \mathrm{pN}$ (circles) and $15.5 \pm 1.5 \mathrm{pN}$ (triangles). The error bar displayed is the "error of the mean", i.e. (standard deviation). $(N)^{-1 / 2}$ where $N$ is the number of data points taken to obtain the average value. Restricting to $1 / \mathrm{C}_{0} \leq 0.02$ $\mu \mathrm{M}^{-1}$, we have plotted linear fits to the data [15]. We obtained $\mathrm{V}_{\max }=129 \pm 8 \mathrm{~b} / \mathrm{s}$, and $\mathrm{K}_{M}(5 \mathrm{pN})=174 \pm 17 \mu \mathrm{M}$, $\mathrm{K}_{M}(11 \mathrm{pN})=213 \pm 22 \mu \mathrm{M}$ and $\mathrm{K}_{M}(15.5 \mathrm{pN})=243 \pm 27$ $\mu \mathrm{M}$, these results are consistent with previous bulk measurements [16]. Number of points: 395; number of enzymes: 96; time of transcription: $1 \mathrm{~h} 50 \mathrm{~min}(1 \mathrm{~h} 10 \mathrm{~min}$ at $5 \mathrm{pN}, 30 \mathrm{~min}$ at $11 \mathrm{pN}$ and $10 \mathrm{~min}$ at $15.5 \mathrm{pN}$ ). Inset: Experimental apparent $\mathrm{K}_{M}$ vs. force: the $\mathrm{K}_{M}$ values are deduced from linear fits. The data is fit by the following expression (see text): $\mathrm{K}_{M}(\mathrm{~F})$ $=\mathrm{K}_{\text {diss }}(1+\mathrm{K} \cdot \exp [\mathrm{F} \delta / \mathrm{kT}])$, where $\delta$ is enzyme step size, a fixed parameter equal to one base pair. The black line is the best fit: one gets $\mathrm{K}=0.27$ and $\mathrm{K}_{\text {diss }}=124 \mu \mathrm{M}$. To show the effect of a change in the parameter $\mathrm{K}$, the other lines are fits obtained when imposing respectively $\ln (\mathrm{K})=0$ and $\ln (\mathrm{K})=-5$, situations where $\mathrm{E}_{\text {post }}$ would either have the same free energy as $\mathrm{E}_{\text {pre }}$, or be more stable by $5 \mathrm{kT}$. mechanism put forward in [4], where it is assumed that (i) the disassociation of $\mathrm{PPi}$ is directly coordinated with translocation and that (ii) a large free energy change is associated with translocation. The structural data suggest that a force opposing forward motion will push the enzyme backward from the post-translocated state. We propose that there is a transient state in the catalytic cycle where PPi is just disassociated, but the enzyme has not yet moved forward (noted $\mathrm{E}_{\text {pre }}$ in Fig. 4). In the absence of PPi [18], a force opposing the forward motion would favor such a transient state. Then a coherent picture emerges, compatible both with structural and kinetic (single molecule) data: competitive inhibition by force derives from the force-induced state-occupancy-bias between $\mathrm{E}_{\text {pre }}$ (unable to bind NTP) and $\mathrm{E}_{\text {post }}$ (able to bind NTP). The enzyme advances after PPi release, that (as a charged molecule as argued in [4]) modifies the preferred state of the enzyme when leaving the active site, in part because the electrostatic interactions are modified: this induces a forward motion. From our single molecule measurements (see Fig. 3), and using the above model, this change of energy $\Delta \mathrm{G}_{0}$ is about $-1.3 \mathrm{kT}$.

What other energetic contributions could there be? The structural data indicates that there is little change in structure upon PPi cleavage and phosphodiester bond formation, but this does not mean that there is no associated energy loss; actually it is likely that some fraction of the global energy loss occurs at this step. Also, it may be argued that between the states $\mathrm{E}_{\text {pre }}: \mathrm{PPi}$ and $\mathrm{E}_{\text {post }}$ there is an "helicase action" [4] of the polymerase, because the transcription bubble is displaced forward: one DNA-DNA base pair is broken, a DNA-DNA base pair is formed, and one DNA-RNA base pair is broken, resulting in a net necessary energy input $\Delta \mathrm{G}_{1}$ that we estimate to be on the average of the order of $\approx 3 \mathrm{kT}$ [11]. Along this view, one arbitrarily separates the energetic cost of the translocation step of the transcribing complex in two
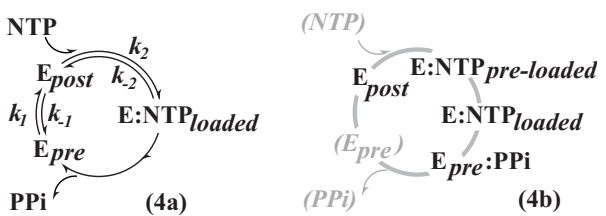

FIG. 4: Reaction cycles. (4a): model inspired by Guajardo and Sousa [17]; $\mathrm{E}_{\text {pre }}$ : pre-translocated enzyme after hydrolysis and PPi release, unable to bind NTP; $\mathrm{E}_{\text {post }}$ : posttranslocated enzyme waiting for NTP binding; E: $\mathrm{NTP}_{\text {loaded }}$ : enzyme with NTP bound in proper position in the catalytic pocket; PPi: pyrophosphate [18]. (4b): states identified from published crystal structures; $\mathrm{E}_{\text {post }}[2,3]$, and E:NTP $\mathrm{N}_{\text {loaded }}[4]$ designate the same states as in $4 \mathrm{a} ; \mathrm{E}: \mathrm{NTP}_{\text {pre-loaded }}$ : posttranslocated complex with NTP pre-loaded [5]; $\mathrm{E}_{\text {pre }}: \mathrm{PPi}$ : enzyme after nucleotide hydrolysis, but before PPi release [4]. $\mathrm{E}_{\text {pre }}$, corresponds to a pre-translocated transient state (without corresponding crystal structure, see text). 
parts, respectively the energy $\Delta G_{2}$ associated with the conformational change of the protein itself (induced after PPi has left), and the energetic change $\Delta \mathrm{G}_{1}$ in DNA conformation. Then $\Delta \mathrm{G}_{2}=-\Delta \mathrm{G}_{1}+\Delta \mathrm{G}_{0} \approx-4 \mathrm{kT}$, which is a sizeable fraction of the energy of hydrolysis. Nevertheless, $\Delta \mathrm{G}_{0}$ appears as the available energy "output" that the transcribing complex as a whole is investing in translocation, and this is what we measure.

The value estimated for $\Delta \mathrm{G}_{0}$ is small, as compared to the free energy of hydrolysis [7]. Therefore, translocation ( $\mathrm{E}_{\text {pre }}$ to $\left.\mathrm{E}_{\text {post }}\right)$ occurs by thermal motion only slightly biased (by $-1.3 \mathrm{kT}$ at zero force). This mechanism of forward motion of the whole transcribing complex may thus be termed a "weak" powerstroke (i.e. in essence close to a Brownian motor description).

It has been shown [21] using the $E$. coli RNAP at high NTPs concentration $(1 \mathrm{mM})$, that force (below $15 \mathrm{pN}$ ) does not affect the elongation rate, a result consistent with our present observations on T7 RNAP. This contrasts with other molecular motors such as kinesin, F1 ATPase, or the Phi 29 portal [22-24] for which it was shown that force slows movement or rotation, even at saturating ATP concentrations. From this point of view, this characteristic of RNA polymerases appears to be different from other molecular motors.

We thank U. Bockelmann, J. Guillerez and M. Dreyfus for participating in the early phase of the project, and for discussions, A. Dawid for his help on the setup of the experimental system and for discussions, R. Sousa for his suggestions and K. Neuman for his critical reading of the manuscript. This work has been funded by CNRS, MJENR and universités Paris VI and Paris VII.

* heslot@lpa.ens.fr

[1] J. Gelles and R. Landick R., Cell 9313 (1998)

[2] T.H. Tahirov et al., Nature 42043 (2002)

[3] Y.W. Yin and T.A. Steitz, Science 2981387 (2002)

[4] Y.W. Yin and T.A. Steitz , Cell 116393 (2004)

[5] D. Temiakov et al., Cell 116381 (2004)

[6] MECHANISMS of TRANSCRIPTION, F. Eckstein and D.M.J. Lilley ed, Springer-Verlag Berlin (1997)

[7] See references [8], the standard Gibbs free energy $\Delta G$ is typically of the order of $-20 \mathrm{kT}$ for breaking NTP to form NMP and $\mathrm{PP}_{i}$, and the $\Delta \mathrm{G}$ for phosphodiesther bond formation during DNA ligation has been estimated to be of the order of $+10 \mathrm{kT}$. In the case of a newly inserted base, there will also be an entropic penalty due to the base immobilization in the elongating chain.

[8] R.A. Alberty J. Biol. Chem. 2443290 (1969) and K.S. Dickson, C.M. Burns and J.P. Richardson J. Biol Chem. 27515828 (2000) and A.S.A. Conceição et al. PNAS 100 14719 (2003).

[9] The initially transcribed sequence is designed to obtain stalled complexes at +15 with respect to the transcription initiation start, by using only three nu- cleotides (ATP, GTP, CTP) at a concentration of 0.4 $\mathrm{mM}$ each. The +15 incorporation requires UTP which is not present. In $5 \mu \mathrm{l}$ of initiation buffer ( $40 \mathrm{mM}$ Trisacetate $\mathrm{pH}$ 7.9, $8 \mathrm{mM}$ Mg-acetate, $1 \mathrm{mM}$ DTT, $0.1 \mathrm{mM}$ EDTA), about $100 \mathrm{nM}$ of biotinylated T7 RNAP is mixed with $1 \mathrm{nM}$ of DNA and about $10^{5}$ beads $(1 \mu \mathrm{m}$ diameter, covered with an antibody against digoxygenin). After an incubation time of 1 minute, the stalled complexes are purified at low speed centrifugation $\left(300 \mathrm{~m} / \mathrm{s}^{2}\right.$ for $1 \mathrm{~min}$ 40 ) in a sucrose gradient. The fraction containing beads with tethered DNA is collected and deposited on the sample (with supplementary $25 \mathrm{mM}$ potassium glutamate): this procedure eliminates the excess of free polymerases, and also reduces the concentration of nucleotides present during initiation, to below $0.5 \mu \mathrm{M}$ for each nucleotide.

[10] The T7 RNA polymerase is biotin-tagged by adding the amino acid sequence MAGGLNDIFEAQ $\underline{K M E W R L E}$ at the $\mathrm{N}$ terminus of the protein (the underlined Lysine corresponds to the biotinylated amino acid). The $\lambda$ DNA substrate can be transcribed by the biotinylated $\mathrm{T} 7$ RNAP over more than $10 \mathrm{~kb}$ in our set-up.

[11] U. Bockelmann et al., Biophys. J. 821537 (2002)

[12] The sequence 5'-ATCTGTT-3', known to induce pause or arrest for the T7 RNAP is present in $\lambda$ DNA but at the end of the genome, in regions never explored in our experiment.

[13] (a) N.R. Forde et al., PNAS 1811682 (2002),

(b) K. Adelman et al., PNAS 9913538 (2002)

(c) KC Neuman et al., Cell 115437 (2003)

[14] (a) Q. Xue and E.S. Yeung Nature 373681 (1995),

(b) H.P. Lu , L. Xun , S. Xie , Science 2821877 (1998)

(c) G.J.L. Wuite et al., Nature 404103 (2000)

(d) SF Tolic-Norrelykke et al., JBC 2793292 (2004)

[15] Data in Fig. 3 have been fitted by linear fits excluding the points at $1 / \mathrm{C}_{0}=0.033 \mu \mathrm{M}^{-1}$, as there could be a possible non-Michaelien effect at very low concentration. Each point is weighted by its error bar (indicated in the figure), and the three fits are performed with $\mathrm{K}_{M}$ and $\mathrm{V}_{\max }$ both as force-dependent parameters with the following condition: $\mathrm{V}_{\max }(5 \mathrm{pN}) \geq \mathrm{V}_{\max }(11 \mathrm{pN}) \geq \mathrm{V}_{\max }$ $(15.5 \mathrm{pN})$. The fit leads to a constant $\mathrm{V}_{\max }$ and a forcedependent $\mathrm{K}_{M}$ with the correlation factor $\mathrm{R}=0.99$.

[16] (a) M. Chamberlin, J. Ring, J. Biol. Chem. 2482235 (1973) (b) G.M. Skinner et al., ibid. 2793239 (2004).

[17] R. Guajardo and R. Sousa, J. Mol. Biol. 2659 (1997)

[18] PPi is released during NTP hydrolysis. No PPi is added to the solution. However PPi is generated as transcription proceeds. Taking the known analytic solution for diffusion from a source (the polymerase taken to be $5 \mathrm{~nm}$ of radius) generating particles ( $\mathrm{PPi}$ ) at a constant rate, the typical local concentration of PPi in the immediate vicinity of the polymerase is estimated to be below 0.03 $\mu \mathrm{M}$ when transcription proceeds at $100 \mathrm{~b} / \mathrm{sec}$.

[19] (a) F. Jülicher, A. Adjari and J. Prost, Rev. Mod. Phys. 691269 (1997)

(b) R.D. Astumian, Science 276917 (1997)

[20] In the "two state" model, it was also considered that the potential in state 2 could be modulated, but its minima are then at the same location as the minima in state 1.

[21] M.D. Wang et al., Science 282902 (1998)

[22] M.J. Schnitzer, K. Visscher and S.M. Block, Nat. Cell Biol. 2718 (2000)

[23] R. Yasuda et al., Nature 410898 (2001)

[24] D.E. Smith et al., Nature 413748 (2001) 